\title{
SUMS OF SQUARES OF COMPLEX VECTOR FIELDS AND (ANALYTIC-) HYPOELLIPTICITY
}

\author{
Antonio Bove, Makhlouf Derridj, Joseph J. Kohn, and David S. \\ TARTAKOFF
}

\begin{abstract}
We prove hypoellipticity with loss of $\frac{k-1}{m}$ derivatives in two ways using different a priori estimates, together with analytic hypoellipticity, for

$$
P_{m, k}^{F}=L_{m}^{F} \overline{L_{m}^{F}}+\overline{L_{m}^{F}}|z|^{2 k} L_{m}^{F}, \quad L_{m}^{F}=\frac{\partial}{\partial z}+i F_{z} \frac{\partial}{\partial t},
$$

for $F(z, \bar{z})$ such that $F_{z \bar{z}}=|z|^{2(m-1)} g, g(0) \neq 0$, generalizing results of [7], [5]. For $F(z, \bar{z})=f\left(|z|^{2}\right)$, the loss is shown to be optimal.
\end{abstract}

\section{Introduction and statement of theorems}

In his recent paper, [7], J. J. Kohn exhibited a sum of squares of complex vector fields which satisfied the bracket condition but which was not subelliptic; nonetheless, he showed that the operator was hypoelliptic, though with a large loss of derivatives. His example was:

$$
P_{k}=L \bar{L}+\bar{L}|z|^{2 k} L=-\bar{L}^{*} \bar{L}-\left(\bar{z}^{k} L\right)^{*} \bar{z}^{k} L \quad \text { with } \quad L=\frac{\partial}{\partial z}+i \bar{z} \frac{\partial}{\partial t} .
$$

The a priori estimate Kohn established is a strong one and in this case (since the operator is independent of the variable $t$,) leads virtually at once to the hypoellipticity of $P_{k}$ : for any $s$, there exists a constant $C_{s}$ such that for all smooth $u$ and any pair of cut-off functions $\varphi, \tilde{\varphi}$ with $\tilde{\varphi} \equiv 1$ near $\operatorname{supp} \varphi$,

$$
\|\varphi u\|_{s}^{2} \leq C_{s}\left\|\tilde{\varphi} P_{k} u\right\|_{s+k-1}^{2}+C_{s}\|u\|_{-\infty}^{2}
$$

Here the last norm stands for a norm of arbitrarily low order, with the constant preceeding it possibly depending on the order of that norm, and $u$ assumed to be of (possibly large) compact support.

Subsequently, in [5], M. Derridj and D. S. Tartakoff proved analytic hypoellipticity for $P_{k}$ using rather different methods, namely they established an inequality for functions $v$ of small support, hence an estimate which did not require explicit cut-off functions, reserving the necessity of localizing an actual solution to a neighborhood of a point to the proof of (analytic) hypoellipticity: for any $s$, there exists a constant $C_{s}$ such that for all $v \in C_{0}^{\infty}$ of small support,

$$
\|v\|_{s-\frac{k-1}{2}}^{2}+\|\bar{L} v\|_{s}^{2}+\left\|\bar{z}^{k} L v\right\|_{s}^{2} \leq C_{s}\left|\left(P_{k} v, v\right)_{s}\right|+C_{s}\|v\|_{-\infty}^{2}
$$

which of course yields the previous estimate at once without the cut-off functions but only for $u$ already known to have (small) compact support.

Received by the editors January 23, 2006. 
This paper was partly motivated by the effort to understand the relationship between these estimates, partly to obtain a simpler (or at least more concise) derivation of the former, and finally to generalize these results where possible.

In [12], Tartakoff had already sharpened the methods of [5] to include the example of the operator

$$
P_{m, k}=L_{m} \overline{L_{m}}+\overline{L_{m}}|z|^{2 k} L_{m} \quad \text { with } \quad L_{m}=\frac{\partial}{\partial z}+i \bar{z}|z|^{2(m-1)} \frac{\partial}{\partial t}
$$

based on the tangential vector fields to a domain in $\mathbb{C}^{2}$ of finite type; the technical work was heavily dependent on the methods of [4].

Both [12] and [5] include proofs of $C^{\infty}$ - hypoellipticity by 'truncating' the proofs of analytic hypoellipticity, hence use the entire machinery that has come to be known as $\left(T^{p}\right)_{\varphi}$ since $[10]$.

In this paper, we consider the more general operator

$$
P_{m, k}^{F}=L_{m}^{F} \overline{L_{m}^{F}}+\overline{L_{m}^{F}}|z|^{2 k} L_{m}^{F} \quad \text { with } \quad L_{m}^{F}=\frac{\partial}{\partial z}+i F_{z} \frac{\partial}{\partial t}
$$

with

$$
F_{z \bar{z}}=|z|^{2(m-1)} g, g(0) \neq 0, F_{z}=\bar{z}|z|^{2(m-1)} h
$$

whose prototype, when $F(z, \bar{z})=|z|^{2 m} / m$, is the operator $P_{m, k}$ discussed above.

Here we establish two families of estimates for $P_{m, k}^{F}$, and prove the optimality of these estimates under the additional restriction

$$
F(z, \bar{z})=f\left(|z|^{2}\right)
$$

We will then use one of the estimates to prove $C^{\infty}$ hypoellipticity with precise loss of $\frac{k-1}{m}$ derivatives and the other to prove $C^{\omega}$ hypoellipticity and to give another proof of $C^{\infty}$ hypoellipticity with the prescribed loss.

A note on the norms used is in order. All of our norms and derivations are done in $L^{2}(z, \bar{z}) \times H^{s}(t)$. There are several reasons for this. First, Proposition 1.1 could, for $s=0$, trivially have the norm on the left replaced with the full $-\frac{k-1}{2 m}$ norm, then as mentioned below, using a cut-off in $\tau$ dual to $t$ which tends to the identity, one can prove easily that since $\partial_{t}$ commutes with $P$, high $t$ derivatives of the solution belong to $H^{s-\frac{m-1}{m}}$ (in $t$ ) provided this is true of $P u$ in $H^{s}$ norm.

But the whole classical theory of pseudo-differential operators and wave front sets allows us to microlocalize the consideration of hypoellipticity. For it is clear that if $z \neq 0$, the operator is elliptic and hence even analytic hypoelliptic, and gains two derivatives. For $z$ close to zero, one must look in the cotangent space, $(z, t ; \zeta, \tau)$ which, in the complement of $(z, t ; 0,0)$ we write as the union of overlapping cones: the cones $\Gamma^{ \pm}$contain $\tau=+1, \zeta=0$ and $\tau=-1, \zeta=0$ respectively, fixing $g(0)>0$ for definiteness, while the "elliptic" cone $\Gamma^{0}$ contains $\tau=0$. In $\Gamma^{0}$, the operator $P$ is also elliptic, since this is true of $L \bar{L}$. In $\Gamma^{-}$, the operator $P$ is maximally hypoelliptic and hence is subelliptic with loss of $1 / 2 m$ derivatives $(\|L v\|$ is bounded by $\|\bar{L} v\|$ there, hence the operator is maximally hypoelliptic, which means that the real and imaginary parts of $L$ and $\bar{L}$ are bounded by $P$, and by Hörmander's condition, subelliptic and one has the estimate of Proposition 1.1 with the $H^{s+\frac{1}{2 m}}$ norm on the left), hence is (microlocally) hypoelliptic with a gain of $\frac{1}{2 m}$ derivatives in that region by conventional arguments. 
It is only in the positive cone that all of this work is necessary, and there in addition to having estimates such as Lemma 2.3 below, we also know (as we would in $\Gamma^{-}$as well) that $|\zeta| \leq C|\tau|$ so that estimating high derivatives in $t$ will yield control in all directions.

In the two Propositions which follow, the notation $A \lesssim B$ will mean that $A \leq C B$ with $C$ uniform in $v \in C_{0}^{\infty}$ and locally so in $s$, and $F$ is assumed to satisfy the conditions of (2.1) above.

Proposition 1.1. For $v$ of small support,

$$
\|v\|_{s-\frac{k-1}{2 m}}^{2}+\left\|\overline{L_{m}^{F}} v\right\|_{s}^{2}+\left\|\bar{z}^{k} L_{m}^{F} v\right\|_{s}^{2} \lesssim\left|\left(P_{m, k}^{F} v, v\right)_{s}\right|+\|v\|_{-\infty}^{2}
$$

Proposition 1.2. For any pair of cut-off functions $\varphi, \tilde{\varphi}$ with $\tilde{\varphi} \equiv 1$ near supp $\varphi$, and for $u$ of support in a fixed (not necessarily small) compact set,

$$
\|\varphi u\|_{s}^{2} \lesssim\left\|\tilde{\varphi} P_{m, k}^{F} u\right\|_{s+\frac{k-1}{m}}^{2}+\|u\|_{-\infty}^{2}
$$

Proposition 1.3. For the case $F(z, \bar{z})=f\left(|z|^{2}\right) b(z, \bar{z}), b(0) \neq 0$ the loss in Propositions 1.1 and 1.2 cannot be improved.

Theorem 1. $P_{m, k}^{F}$ is locally hypoelliptic with loss of $\frac{k-1}{m}$ derivatives: $P_{m, k}^{F} u \in$ $H^{s} \Longrightarrow u \in H^{s-\frac{k-1}{m}}$.

Remark 1. In [3], Christ showed that for Kohn's original example the addition of a pure second derivative in a new variable destroyed hypoellipticity altogether, and we would be surprised if a similar phenomenon did not obtain in the present generality.

\section{Preliminary observations and lemmas}

The first observation concerns the apparent difference between the two a priori estimates in the two Propositions above and their use. The second estimate explicitly introduces a second cut-off function, although, as we shall see, except for the last term, the function $\tilde{\varphi}$ may be replaced by certain derivatives of $\varphi$. That is, except for a norm of sufficiently low order, we may control the terms on the right by derivatives of the given localizing function. In fact, the same is true in the proof of (analytic) hypoellipticity using the first estimate - we proceed with a balanced localization $\left(T^{p}\right)_{\varphi}$ of high derivatives in $T=\partial_{t}$ and encounter errors expressed as derivatives of the localizing function we start with and then at a certain point (in this case a fraction of the derivatives we seek to estimate), we are forced to introduce a cut-off function with strictly larger support and to construct a whole new balanced sum $\left(T^{\tilde{p}}\right)_{\tilde{\varphi}}$ around this new localizing function - and for the analyticity proof we need to control these supports in a very precise way.

It is not at all clear how to pass from one setting to the other - neither estimate trivially implies the other and the proofs of hypoellipticity are not trivially comparable, but they do seem to contain the same elements.

Our first technical observation concerns the dependence of localizing functions on $z$. In order to localize to a neighborhood of 0 , we may take a product of a function of $z, \bar{z}$ of small support but identically equal to one near the origin in $\mathbb{C}$ with another function of $t$ only, again taken to be of small support. Whenever the first of these functions is differentiated, the resulting function is supported away from $z=0$, hence 
in a region where the operator $P$ is in fact subelliptic and hence far better behaved. We shall ignore such regions and thus take all localizations to be functions of $t$ only.

To make the proofs of Propositions 1.1 and 1.2 flow more smoothly, we prepare some easy lemmas which will be used repeatedly in the sequel. By integration by parts and shifting powers of $z$ from one side of an inner product to the other, these lemmas, especially Lemma 2.4 and 2.5, which are often used, express the obvious fact that by grouping one power of $z$ and a fractional power of $\Lambda_{t}$, effectively a fractional power of $\partial_{t}$, as a unit, say $A=z \Lambda_{t}^{\rho}$, one may move powers of $A$ from one side of an inner product to the other. In all of these lemmas, $w$ will denote a smooth function of (small) compact support and the superscript ' + ' will indicate that the function has been microlocalized to the positive cone for the symbol of $\partial_{t}$.

The following estimates are locally uniform in $s$, and here and in the sequel the notations "s.c." and "l.c." denote "arbitrary small positive constant" and "large constant" (depending on the small constant).

Furthermore, from now on we will write $L$ for $L_{m}^{F}, \bar{L}$ for $\overline{L_{m}^{F}}$, and $P$ for $P_{m, k}^{F}$.

Lemma 2.1. $\|L w\|_{s-1 / 2}^{2} \lesssim\|\bar{L} w\|_{s-1 / 2}^{2}+\left\|z^{m-1} w\right\|_{s}$.

Proof. Integration by parts since $[L, \bar{L}]=-2 i F_{z \bar{z}} \partial_{t},\left|F_{z \bar{z}}\right| \lesssim|z|^{2(m-1)}$.

Lemma 2.2. $\left\|z^{m-1} w\right\|_{s+1 / 2}^{2} \lesssim\|L w\|_{s}^{2}+\|\bar{L} w\|_{s}^{2}$.

Proof. Integration by parts since $[L, \bar{L}]=-2 i F_{z \bar{z}} \partial_{t}$ and $F_{z \bar{z}} \geq c|z|^{2(m-1)}$.

Lemma 2.3. $\left\|\bar{L} w^{+}\right\|_{s}+\left\|z^{m-1} w^{+}\right\|_{s+1 / 2}^{2} \lesssim\left\|L w^{+}\right\|_{s}+\|w\|_{s}$.

Proof. The same identity where the symbol of $-2 i \partial_{t}$ has the appropriate sign.

Lemma 2.4. $\left\|z^{r} w\right\|_{\mu} \leq$ s.c. $\left\|z^{r-n_{1}} w\right\|_{\mu-n_{1} \rho}^{2}+$ l.c. $\left\|z^{r+n_{2}} w\right\|_{\mu+n_{2} \rho}^{2}, n_{1} \leq r$.

Proof. Let $A=z \Lambda_{t}^{\rho}$. Then for example

$$
\left\|A^{r} w\right\|_{\mu}^{2}=\left(A^{r-n_{1}} w, A^{r+n_{1}}\right)_{\mu} \leq \text { s.c. }\left\|A^{r-n_{1}} w\right\|_{\mu}^{2}+\text { l.c. }\left\|A^{r+n_{1}} w\right\|_{\mu}^{2}
$$

but then the second of these terms may be related to lower and higher powers of $A$, and the result follows.

Lemma 2.5. $\left\|z^{r} w\right\|_{\mu} \leq$ l.c. $\left\|z^{r-n_{1}} w\right\|_{\mu-n_{1} \rho}^{2}+$ s.c. $\left\|z^{r+n_{2}} w\right\|_{\mu+n_{2} \rho}^{2}, n_{1} \leq r$.

Proof. Completely analogous.

Lemma 2.6. $\|w\|_{0}^{2} \lesssim\|z \bar{L} w\|_{0}^{2}+\|z L w\|_{0}^{2}$.

Proof. This is the subelliptic multiplier argument:

$$
\begin{aligned}
\|w\|_{0}^{2} & =|([L, z] w, w)| \leq|(w, \bar{z} \bar{L} w)|+|(z L w, w)| \\
& \leq \text { s.c. }\|w\|_{0}^{2}+\text { l.c. }\left(\|z \bar{L} w\|_{0}^{2}+\|z L w\|_{0}^{2}\right) .
\end{aligned}
$$

Lemma 2.7. $\|\varphi u\|_{0}^{2} \lesssim\|z \bar{L} \varphi u\|_{0}^{2}+\|\bar{z} L \varphi u\|_{0}^{2}$.

Proof. This is the previous lemma with $w=\varphi u$.

Lemma 2.8. $\|\varphi u\|_{0}^{2} \lesssim\|z \varphi \bar{L} u\|_{0}^{2}+\|\bar{z} \varphi L u\|_{0}^{2}+\left\|z^{2 m} \varphi^{\prime} u\right\|_{0}^{2}$. 
Proof. This is just the observation that $|[L, \varphi] u| \sim|z|^{2 m-1}\left|\varphi_{t}\right|$.

Lemma 2.9. $\left\|z \varphi^{\prime} u\right\|_{0}^{2} \lesssim\left\|\bar{L} z \varphi^{\prime} u\right\|_{-\frac{1}{2 m}}^{2}+\left\|L z \varphi^{\prime} u\right\|_{-\frac{1}{2 m}}^{2}+\left\|z \varphi^{\prime} u\right\|_{-\frac{1}{2 m}}^{2}$

Proof. This is just the observation that the vector fields $L$ and $\bar{L}$, or rather their real and imaginary parts, satisfy the (real) bracket condition and hence form a subelliptic system in the usual sense with $\varepsilon=1 / 2 m$, and then the whole subelliptic estimate is lowered by $1 / 2 m$.

\section{Proof of Proposition 1.1}

To prove Proposition 1.1, the a priori estimate on compactly supported functions, we set $r=-\frac{k-1}{2 m}$ and $\tau=\frac{1}{2 m}$. Note that $r$ need not be negative, but $r-\tau=-\frac{k}{2 m} \leq 0$. Then we have, since $r \leq \tau$ :

$$
\begin{aligned}
\|v\|_{r}^{2} & =\left((L z) \Lambda_{t}^{r} v, \Lambda_{t}^{r} v\right)=\left(z \Lambda_{t}^{2 r} v, \bar{L}_{m} v\right)-\left(L \Lambda_{t}^{r-\tau} v, \bar{z} \Lambda_{t}^{r+\tau} v\right) \\
& \leq C\left\{\|\bar{L} v\|_{0}^{2}+\text { s.c. }\left\|L \Lambda_{t}^{r-\tau} v\right\|_{0}^{2}+\text { l.c. }\left\|z \Lambda_{t}^{\tau}\left(\Lambda_{t}^{r} v\right)\right\|_{0}^{2}\right\} \\
& \leq C\left\{\|\bar{L} v\|_{0}^{2}+\text { l.c. }\left\|z \Lambda_{t}^{\tau}\left(\Lambda_{t}^{r} v\right)\right\|_{0}^{2}+\text { s.c. }\left\|z^{m-1} \Lambda_{t}^{\frac{1}{2}-\tau}\left(\Lambda_{t}^{r} v\right)\right\|_{0}^{2}\right\} \\
& \leq C\left\{\|\bar{L} v\|_{0}^{2}+\text { l.c. }\left\|z \Lambda_{t}^{\tau}\left(\Lambda_{t}^{r} v\right)\right\|_{0}^{2}+\text { s.c. }\left\|z^{m-1} \Lambda_{t}^{(m-1) \tau}\left(\Lambda_{t}^{r} v\right)\right\|_{0}^{2}\right\} .
\end{aligned}
$$

When $m=1$, this last term is just s.c. $\|v\|_{r}^{2}$ but for $0<a \leq m-1+k$, we use Lemma 2.4 in the form

$$
\begin{aligned}
\left\|z^{a} \Lambda_{t}^{a \tau} w\right\|_{0}^{2} & \leq \text { s.c. }\|w\|_{0}^{2}+\text { l.c. }\left\|z^{m-1+k} \Lambda_{t}^{(m-1+k) \tau} w\right\|_{0}^{2} \\
& =\text { s.c. }\|w\|_{0}^{2}+\text { l.c. }\left\|z^{m-1+k} \Lambda_{t}^{\frac{1}{2}}\left(\Lambda_{t}^{(m-1+k) \tau-\frac{1}{2}} w\right)\right\|_{0}^{2}
\end{aligned}
$$

with $w=\Lambda_{t}^{r} v$ twice, once for $a=1$ and once for $a=m-1$.

Inserting this in the estimate above for $\|v\|_{r}^{2}$, we find

$$
\begin{aligned}
\|v\|_{r}^{2} & \leq C\left\{\|\bar{L} v\|_{0}^{2}+\left\|z^{m-1+k} \Lambda_{t}^{\frac{1}{2}} \Lambda_{t}^{(m-1+k) \tau-\frac{1}{2}+r} v\right\|_{0}^{2}\right\} \\
& =C\left\{\|\bar{L} v\|_{0}^{2}+\left\|z^{m-1+k} \Lambda_{t}^{\frac{1}{2}} v\right\|_{0}^{2}\right\}
\end{aligned}
$$

since $(m-1+k) \tau-\frac{1}{2}+r=0$.

On the other hand, we have by Lemma 2.2,

$$
\left\|z^{m-1+k} \Lambda_{t}^{\frac{1}{2}} v\right\|_{0}^{2} \leq C\left\{\|\bar{L} v\|_{0}^{2}+\left\|\bar{z}^{k} L v\right\|_{0}^{2}\right\},
$$

(which one proves from the Lemma with the additional term $\left\|z^{k-1} v\right\|_{0}^{2}$ on the right and then, writing $z^{k-1} \sim\left[L, z^{k}\right]$, absorbs this term by the other two). Thus we arrive at

$$
\|v\|_{r}^{2} \leq C\left\{\|\bar{L} v\|_{0}^{2}+\left\|\bar{z}^{k} L v\right\|_{0}^{2}\right\}=C|(P v, v)| \leq l . c .\|P v\|_{-r}^{2}+\text { s.c. }\|v\|_{r}^{2}
$$

or

$$
\|v\|_{-\frac{k-1}{2 m}}^{2}+\|\bar{L} v\|_{0}^{2}+\left\|\bar{z}^{k} L v\right\|_{0}^{2} \lesssim C\|P v\|_{\frac{k-1}{2 m}}^{2}, v \in C_{0}^{\infty},
$$




\section{Proof of Proposition 1.2. Case $k=1$}

For $k=1$ we will establish the estimate (for $u$ of small support near $z=0$ ) using only Lemmas 2.3 and 2.8:

$$
\begin{aligned}
\|\varphi u\|_{s}^{2}+\|\varphi \bar{L} u\|_{s}^{2}+\|\bar{z} \varphi L u\|_{s}^{2} & \equiv \sum_{j=1}^{3}(L H S)_{j} \\
& \lesssim \sum_{j=0}^{N}\left\|\varphi^{(j)} P u\right\|_{s-j / 2}^{2}+\left\|\varphi^{(N)} u\right\|_{s-N / 2}^{2}+\|u\|_{-\infty}^{2} .
\end{aligned}
$$

Here and elsewhere, we will find the following definition useful:

Definition 4.1. The designation "RJ" (for "Relative Junk") will apply to any multiple of any of the terms $(L H S)_{j}$ that we are in the process of estimating but with lower Sobolev index and possibly a derivative on the localizing function - in other words, to a term which will be iteratively estimated at the end.

For any value of $k$, from Lemma 2.8,

$$
(L H S)_{1} \equiv\|\varphi u\|_{0}^{2} \lesssim\|z \varphi \bar{L} u\|_{0}^{2}+\|\bar{z} \varphi L u\|_{0}^{2}+\left\|z^{m} \varphi^{\prime} u\right\|_{0}^{2}
$$

(Lemma 2.8 even gives $z^{2 m}$ in place of $z^{m}$ ). We claim that this last term is RJ. To see this, Lemma 2.3 tells us that

$$
\left\|z^{m} \varphi^{\prime} u\right\|_{0}^{2}=\left\|z^{m-1} \bar{z} \varphi^{\prime} u\right\|_{0}^{2} \lesssim\left\|\bar{z} L \varphi^{\prime} u\right\|_{-1 / 2}^{2}+\left\|z \varphi^{\prime} u\right\|_{-1 / 2}^{2}=R J
$$

(provided, as we will show, that we can estimate $\|\varphi u\|_{0}^{2}$ and $\|\bar{z} L \varphi u\|_{0}^{2}$ ). Actually, in the next section we will see even that $\left\|z \varphi^{\prime} u\right\|_{0}^{2} \in R J$.

So we have, modulo $R J$,

$$
\begin{aligned}
(L H S)_{1} & \lesssim(L H S)_{2}+(L H S)_{3} \\
& \equiv\|\bar{z} \varphi L u\|_{0}^{2}+\|\varphi \bar{L} u\|_{0}^{2} \\
& =\left|-\left(\bar{L} \varphi^{2}|z|^{2} L u, u\right)-\left(L \varphi^{2} \bar{L} u, u\right)\right| \\
& \lesssim\left|\left(\varphi^{2} P u, u\right)\right|+\left|\left(F_{z} \varphi \varphi^{\prime} \bar{L} u, u\right)\right|+\left|\left(F_{\bar{z}} \varphi \varphi^{\prime}|z|^{2} L u, u\right)\right|+\left|\left(\varphi^{2} \bar{z} L u, u\right)\right| .
\end{aligned}
$$

These last two terms are easy to handle:

$$
\left|\left(F_{z} \varphi \varphi^{\prime} \bar{L} u, u\right)\right|+\left|\left(F_{\bar{z}} \varphi \varphi^{\prime} L u, u\right)\right| \lesssim \text { s.c. }\|\varphi \bar{L} u\|_{0}^{2}+\text { l.c. }\left\|z^{2 m-1} \varphi^{\prime} u\right\|_{0}^{2}+\text { s.c. }\|\bar{z} L u\|_{0}^{2}
$$

which are absorbed modulo the term $\left\|z^{2 m-1} \varphi^{\prime} u\right\|$ which is RJ since $2 m-1 \geq m$.

Thus in all, in the positive cone, with $\tilde{\varphi} \equiv 1$ near the support of $\varphi$, and for any $N$,

$$
\|\varphi u\|_{s}^{2}+\|\varphi \bar{L} u\|_{s}^{2}+\|\bar{z} \varphi L u\|_{s}^{2} \lesssim \sum_{j=0}^{N}\left\|\varphi^{(j)} P u\right\|_{s-j / 2}^{2}+\left\|\varphi^{(N)} u\right\|_{s-N / 2}^{2}+\|u\|_{-\infty}^{2} .
$$

Or, with $\tilde{\varphi} \equiv 1$ near the support of $\varphi$,

$$
\|\varphi u\|_{s}^{2} \lesssim\|\tilde{\varphi} P u\|_{s}^{2}+\|u\|_{-\infty}^{2}
$$




\section{Proof of Proposition 1.2. Case $k>1$}

To prove Proposition 1.2 when $k>1$, which is harder, we cannot just use Proposition 1.1 with a cutoff function $\varphi$ in front of $v$ and then express the right hand side in terms of $(\varphi P v, \varphi v)$ modulo acceptable errors, since bracket of $P$ with $\varphi$ introduces errors easily absorbed only when the basic estimate is subelliptic, which here means $k=0$, the well-known case, or at least, in Kohn's terminology, 'no loss, no gain', namely the case $k=1$ which we just considered.

Instead, we proceed as follows. We will establish again the class of "Relative Junk Terms", denoted $R J$, which are of the same form as those terms being estimated but of lower Sobolev degree, and the localizing function(s) may have received a derivative. These will be treated recursively at the end, in a very simple manner, but to see that a term is RJ one may have to compare it to all eight terms below.

The terms we want to estimate are eight in number, and will be referred to as $(L H S)_{j}, j=1, \ldots 8$. In estimating some the others will occur, generally with small constants, but we set up a generic sum with unknown coefficients $\sum_{j=1}^{j=8} C_{j}(L H S)_{j}$. Specifically, we will establish, for suitable $C_{j}$ to be determined relative to one another,

$$
\begin{array}{r}
C_{1}\|\varphi u\|_{0}^{2}+C_{2}\|\bar{z} \varphi L u\|_{0}^{2}+C_{3}\|\varphi \bar{L} u\|_{\frac{k-1}{2 m}}^{2}+C_{4}\left\|\varphi \bar{z}^{k} L u\right\|_{\frac{k-1}{2 m}}^{2}+C_{5}\left\|z^{2 k+m-2} \varphi u\right\|_{\frac{k-1}{m}}^{2} \\
+C_{6}\left\|\varphi z^{2 k-1} L u\right\|_{\frac{k-1}{m}-\frac{1}{2}}^{2}+C_{7}\left\|z^{m-1} \varphi \bar{L} u\right\|_{\frac{k-1}{m}}^{2}+C_{8}\|L \varphi \bar{L} u\|_{\frac{k-1}{m}-\frac{1}{2}}^{2} \\
=E=\sum_{1}^{8} C_{j}(L H S)_{j} \leq C_{9}\|\varphi P u\|_{\frac{k-1}{m}}^{2}+R J .
\end{array}
$$

In proving $(*)$ we will encounter errors from microlocalization, errors which are supported in regions where the regularity is well understood. As these will be included in $R J$ in any case, we will omit explicit mention of terms of the form $\|u\|_{-\infty}^{2}$.

5.1. Estimating $(L H S)_{1}$. Using Lemma 2.8 and then Lemmas 2.9 and 2.4:

$$
\begin{aligned}
(L H S)_{1} & \equiv\|\varphi u\|_{0}^{2} \\
& \lesssim\|\varphi \bar{L} u\|_{0}^{2}+\|\bar{z} \varphi L u\|_{0}^{2}+\left\|z \varphi^{\prime} u\right\|_{0}^{2} \\
& \lesssim\|\varphi \bar{L} u\|_{0}^{2}+\text { l.c. }\left\|\bar{z}^{k} \varphi L u\right\|_{\frac{k-1}{2 m}}^{2}+\text { s.c. }\|\varphi L u\|_{-\frac{1}{2 m}}^{2}+\left\|z \varphi^{\prime} u\right\|_{0}^{2} .
\end{aligned}
$$

For the third term we write, using Lemma 2.1:

$$
\|\varphi L u\|_{-\frac{1}{2 m}}^{2} \lesssim\left\|z^{m-1} \varphi u\right\|_{-\frac{1}{2 m}+\frac{1}{2}}^{2}+\|\varphi \bar{L} u\|_{-\frac{1}{2 m}}^{2}+\left\|\varphi^{\prime} u\right\|_{-\frac{1}{2 m}}^{2}
$$

(the last two terms are RJ) and from Lemma 2.4,

$$
\left\|z^{m-1} \varphi u\right\|_{-\frac{1}{2 m}+\frac{1}{2}}^{2} \lesssim \text { s.c. }\|\varphi u\|_{0}^{2}+\text { l.c. }\left\|z^{m-1} \bar{z}^{k} \varphi u\right\|_{\frac{1}{2}+\frac{k-1}{2 m}}^{2}
$$

and by Lemma 2.3,

$$
\left\|z^{m-1} \bar{z}^{k} \varphi u\right\|_{\frac{1}{2}+\frac{k-1}{2 m}}^{2} \lesssim\left\|\bar{z}^{k} \varphi L u\right\|_{\frac{k-1}{2 m}}^{2}+\left\|\bar{z}^{k} \varphi u\right\|_{\frac{k-1}{2 m}}^{2}+\left\|z^{k+2 m-1} \varphi^{\prime} u\right\|_{\frac{k-1}{2 m}}^{2} .
$$

Now

$$
\left\|\bar{z}^{k} \varphi u\right\|_{\frac{k-1}{2 m}}^{2} \leq \text { l.c. }\|\varphi u\|_{-\frac{1}{2 m}}^{2}+\text { s.c. }\left\|z^{m-1} \bar{z}^{k} \varphi u\right\|_{\frac{1}{2}+\frac{k-1}{2 m}}^{2}
$$


$=\mathrm{RJ}$ plus a term which can be absorbed by the previous left hand side and a direct application of Lemma 2.4 yields

Lemma 5.1.

$$
\left\|z^{k+2 m-1} \varphi^{\prime} u\right\|_{\frac{k-1}{2 m}}^{2} \lesssim\left\|z^{2 m} \varphi^{\prime} u\right\|_{0}^{2}+\left\|z^{2 k+m-2} \varphi^{\prime} u\right\|_{\frac{k-1}{m}-\frac{1}{2}}^{2}=R J
$$

Putting these together,

$$
\sum_{j=1}^{j=2}(L H S)_{j} \lesssim(L H S)_{3}+(L H S)_{4}+R J
$$

5.2. Estimation of $(L H S)_{3}$ and $(L H S)_{4}$. Using the fact that $\left|F_{z}\right| \sim|z|^{2 m-1}$, we have

$$
\begin{aligned}
(L H S)_{3}+(L H S)_{4}= & \|\varphi \bar{L} u\|_{\frac{k-1}{2 m}}^{2}+\left\|\varphi \bar{z}^{k} L u\right\|_{\frac{k-1}{2 m}}^{2} \\
= & (\varphi P u, \varphi u)_{\frac{k-1}{2 m}}-\left(\left[\bar{L}, \varphi^{2}\right]|z|^{2 k} L u, u\right)_{\frac{k-1}{2 m}}-\left(\left[\varphi^{2}, L\right] \bar{L} u, u\right)_{\frac{k-1}{2 m}} \\
\leq & \text { l.c. }\|\varphi P u\|_{\frac{k-1}{m}}^{2}+\text { s.c. }(L H S)_{1} \\
& +2\left|\left(F_{\bar{z}} \varphi \varphi^{\prime}|z|^{2 k} L u, u\right)_{\frac{k-1}{2 m}}\right|+2\left|\left(F_{z} \varphi \varphi^{\prime} \bar{L} u, u\right)_{\frac{k-1}{2 m}}\right| \\
\leq & \text { l.c. }\|\varphi P u\|_{\frac{k-1}{m}}^{2}+\text { s.c. }(L H S)_{1}+\left\{\text { s.c. }\left\|\varphi \bar{z}^{k} L u\right\|_{\frac{k-1}{2 m}}^{2}\right. \\
& \left.+ \text { l.c. }\left\|z^{2 m-1+k} \varphi^{\prime} u\right\|_{\frac{k-1}{2 m}}^{2}\right\}+\left\{\text { s.c. }\left\|\varphi z^{m-1} \bar{L} u\right\|_{\frac{k-1}{m}}^{2}\right. \\
& \left.+ \text { l.c. }\left\|z^{m} \varphi^{\prime} u\right\|_{0}^{2}\right\}+R J \\
\lesssim & \text { l.c. }\|\varphi P u\|_{\frac{k-1}{m}}^{2}+\text { s.c. }\left\{(L H S)_{1}+(L H S)_{4}+(L H S)_{7}\right\}+R J
\end{aligned}
$$

using Lemma 5.1 since the right hand side (4.2) is $R J$ for any $k$. Thus,

$$
\sum_{1}^{4} C_{j}(L H S)_{j} \leq \text { s.c. }(L H S)_{7}+C_{9}\|\varphi P u\|_{\frac{k-1}{m}}^{2}+R J .
$$

5.3. Estimation of $(L H S)_{5}$ and $(L H S)_{6}$. Setting $\sigma=\frac{k-1}{m}-\frac{1}{2}$ and using Lemma 2.3 ,

$$
\begin{aligned}
(L H S)_{5}+(L H S)_{6} & \equiv\left\|z^{2 k+m-2} \varphi u\right\|_{\frac{k-1}{m}}^{2}+\left\|\varphi z^{2 k-1} L u\right\|_{\sigma}^{2} \\
& \lesssim\left\|\varphi z^{2 k-1} L u\right\|_{\sigma}^{2}+\left\|\varphi z^{2 k+m-3} u\right\|_{\sigma}^{2}+\left\|z^{2 k+3 m-3} \varphi^{\prime} u\right\|_{\sigma}^{2}+R J \\
& \lesssim\left\|\varphi z^{2 k-1} L u\right\|_{\sigma}^{2}+\text { s.c. }\left\|\varphi z^{2 k+2 m-3} u\right\|_{\frac{k-1}{m}}^{2}+\|\varphi u\|_{-\frac{2 m-1}{2 m}}^{2}+R J \\
& \lesssim\left\|\varphi z^{2 k-1} L u\right\|_{\sigma}^{2}+\text { s.c. }(L H S)_{5}+R J
\end{aligned}
$$

(since $2 k+2 m-3 \geq 2 k+m-2$ ) with Lemma 2.4.

So we have to consider

$$
\begin{aligned}
(L H S)_{6} \equiv & \left\|\varphi z^{2 k-1} L u\right\|_{\sigma}^{2} \lesssim\left|\left(\bar{L} \varphi^{2}|z|^{2(2 k-1)} L u, u\right)_{\sigma}\right| \\
& +\left\|\varphi^{\prime} z^{2(k+m-1)} u\right\|_{\sigma}^{2}+\left\|z^{2 k-2} \varphi u\right\|_{\sigma}^{2} \\
\lesssim & \left|\left(\bar{L} \varphi^{2}|z|^{2(2 k-1)} L u, u\right)_{\sigma}\right|+\text { s.c. }\left\{(L H S)_{1}+(L H S)_{5}\right\}+R J
\end{aligned}
$$

by Lemma 2.4 . 
Now for the inner product we have, again using Lemma 2.4,

$$
\begin{aligned}
\left|\left(\bar{L} \varphi^{2}|z|^{2(2 k-1)} L u, u\right)_{\sigma}\right| \leq & \left|\left(2 \varphi \varphi^{\prime} F_{\bar{z}}|z|^{2(2 k-1)} L u, u\right)_{\sigma}\right| \\
& +\left|\left(\varphi \bar{L}|z|^{2 k} L u, z^{2 k-2} \varphi u\right)_{\sigma}\right|+\left|\left(\varphi|z|^{4 k-3} L u, \varphi u\right)_{\sigma}\right| \\
\lesssim & \text { s.c. }\left\|z^{2 k-1} L u\right\|_{\sigma}^{2}+\text { l.c. }\left\|z^{2 k+2 m-2} u\right\|_{\sigma}^{2}+\|\varphi P u\|_{\sigma}^{2} \\
& +\left\|z^{2 k-2} \varphi u\right\|_{\sigma}^{2}+\left|\left(\varphi L \bar{L} u, z^{2 k-2} \varphi u\right)_{\sigma}\right|+\text { s.c. }\left\|z^{2 k-1} \varphi L u\right\|_{\sigma}^{2}
\end{aligned}
$$

so

$$
\begin{aligned}
\left|\left(\bar{L} \varphi^{2}|z|^{2(2 k-1)} L u, u\right)_{\sigma}\right| \lesssim & \text { s.c. }\left\{(L H S)_{6}+(L H S)_{5}+(L H S)_{1}\right\} \\
& +\|P u\|_{\sigma}^{2}+\left|\left(\varphi L \bar{L} u, z^{(2 k-2)} \varphi u\right)_{\sigma}\right|+R J \\
\leq & \text { s.c. }\left\{(L H S)_{6}+(L H S)_{5}+(L H S)_{1}+(L H S)_{8}\right\} \\
& +\|P u\|_{\sigma}^{2}+R J .
\end{aligned}
$$

Thus, so far,

$$
\sum_{1}^{6} C_{j}(L H S)_{j} \leq \text { s.c. }\left\{(L H S)_{7}+(L H S)_{8}\right\}+C_{9}\|\varphi P u\|_{\frac{h-1}{m}}^{2}+R J
$$

but we still need to estimate both $(L H S)_{7}$ and $(L H S)_{8}$ since the definition of $R J$ requires it.

5.4. Estimation of $(L H S)_{7}$ and $(L H S)_{8}$. We proceed to estimate (a small multiple of) the following expression $B$, noting that $\|\varphi L \bar{L} u\|_{\sigma}^{2}=\|L \varphi \bar{L} u\|_{\sigma}^{2}+R J$ :

$$
\begin{aligned}
B & =\left\|z^{m-1} \varphi \bar{L} u\right\|_{\frac{k-1}{m}}^{2}+\|\varphi \bar{L} \bar{L} u\|_{\sigma}^{2}\left(+\|\varphi L \bar{L} u\|_{\sigma}^{2}\right) \\
& \lesssim\|\varphi L \bar{L} u\|_{\sigma}^{2}+R J \\
& \lesssim\left|(\varphi \bar{L} L \bar{L} u, \varphi \bar{L} u)_{\sigma}\right|+\left|\left(\varphi L \bar{L} u, \varphi^{\prime} F_{z} \bar{L} u\right)_{\sigma}\right|+R J \\
& =\left|(\varphi \bar{L} L \bar{L} u, \varphi \bar{L} u)_{\sigma}\right|+\text { s.c.B }+R J \\
& \lesssim\left|(\varphi \bar{L} P u, \varphi \bar{L} u)_{\sigma}\right|+\left|\left(\varphi \bar{L} \bar{L}|z|^{2 k} L u, \varphi \bar{L} u\right)_{\sigma}\right|+\text { s.c.B }+R J \\
& =B_{1}+B_{2}+\text { s.c.B }+ \text { RJ. }
\end{aligned}
$$

Now

$$
\begin{aligned}
B_{1} & =\left|(\varphi \bar{L} P u, \varphi \bar{L} u)_{\sigma}\right| \\
& \leq\left|(\varphi P u, \varphi L \bar{L} u)_{\sigma}\right|+\left|\left(F_{\bar{z}} \varphi \varphi^{\prime} P u, \bar{L} u\right)_{\sigma}\right| \\
& \lesssim\|\varphi P u\|_{\sigma}^{2}+\text { s.c. } B+R J
\end{aligned}
$$

while

$$
\begin{aligned}
B_{2} & =\left|\left(\varphi \bar{L} \bar{L}|z|^{2 k} L u, \varphi \bar{L} u\right)_{\sigma}\right| \\
& \lesssim\left|\left(\varphi \bar{L}|z|^{2 k} \bar{L} L u, \varphi \bar{L} u\right)_{\sigma}\right|+\left|\left(\varphi \bar{L} z|z|^{2(k-1)} L u, \varphi \bar{L} u\right)_{\sigma}\right| \\
& =B_{21}+B_{22} .
\end{aligned}
$$

For $B_{21}$ we have

$$
\begin{aligned}
B_{21} & \lesssim\left|\left(\varphi \bar{L}|z|^{2 k} L \bar{L} u, \varphi \bar{L} u\right)_{\sigma}\right|+\left|\left(\varphi \bar{L}|z|^{2 k} F_{z \bar{z}} T u, \varphi \bar{L} u\right)_{\sigma}\right| \\
& =B_{211}+B_{212}
\end{aligned}
$$


with

$$
\begin{aligned}
B_{211} & \lesssim\left|\left(\varphi|z|^{2 k} L \bar{L} u, L \varphi \bar{L} u\right)_{\sigma}\right|+\left|\left(F_{\bar{z}} \varphi^{\prime}|z|^{2 k} L \bar{L} u, \varphi \bar{L} u\right)_{\sigma}\right| \\
& \leq \text { s.c.B }+ \text { RJ. }
\end{aligned}
$$

For $B_{212}$, the bracket $\left[\bar{L},|z|^{2 k} F_{z \bar{z}}\right]$ will enter. This will contain a factor of the form $z|z|^{2(k-1)+2(m-1)}$, and thus we may move $\bar{z}^{m-1}$ to the right hand side of the inner product leaving a function $g(z) z|z|^{2(k-1)} z^{m-1}$ on the left, for a suitable function $g$. We have

$$
\begin{aligned}
B_{212}= & \left|\left(\varphi \bar{L}|z|^{2 k} F_{z \bar{z}} T u, \varphi \bar{L} u\right)_{\sigma}\right| \\
\lesssim & \left|\left(\varphi \bar{L} F_{z \bar{z}}|z|^{2 k} u, \varphi \bar{L} u\right)_{\frac{k-1}{m}}\right|+\left|\left(\varphi^{\prime} \bar{L} F_{z \bar{z}}|z|^{2 k} u, \varphi \bar{L} u\right)_{\sigma}\right| \\
\lesssim & \left|\left(\varphi F_{z \bar{z}}|z|^{2 k} \bar{L} u, \varphi \bar{L} u\right)_{\frac{k-1}{m}}\right|+\left|\left(\varphi g(z) z|z|^{2(k-1)} z^{m-1} u, \varphi z^{m-1} \bar{L} u\right)_{\frac{k-1}{m}}\right| \\
& +\left|\left(\varphi F_{z \bar{z}}|z|^{2 k} \bar{L} u, \varphi^{\prime} \bar{L} u\right)_{\sigma}\right|+\left|\left(\varphi g(z) z|z|^{2(k-1)} z^{m-1} u, \varphi^{\prime} z^{m-1} \bar{L} u\right)_{\sigma}\right| \\
\lesssim & \text { s.c.B }+R J+\left\|\varphi z^{2 k+m-2} u\right\|_{\frac{k-1}{m}}^{2} \\
\lesssim & \text { s.c.B }+(L H S)_{5}+R J
\end{aligned}
$$

Finally, for $B_{22}$ we have

$$
\begin{aligned}
B_{22} & =\left|\left(\varphi \bar{L} z|z|^{2(k-1)} L u, \varphi \bar{L} u\right)_{\sigma}\right| \\
& \lesssim\left|\left(\varphi z|z|^{2(k-1)} L u, L \varphi \bar{L} u\right)_{\sigma}\right|+\left|\left(\varphi F_{\bar{z}} z|z|^{2(k-1)} L u, \varphi^{\prime} \bar{L} u\right)_{\sigma}\right| \\
& \lesssim\left\|\varphi z^{2 k-1} L u\right\|_{\sigma}^{2}+\text { s.c.B }+ \text { RJ }=(L H S)_{6}+\text { s.c. } B+R J .
\end{aligned}
$$

Thus we have

$$
\text { s.c.B } \lesssim s . c .\left\{(L H S)_{1}+(L H S)_{4}+(L H S)_{7}+(L H S)_{6}\right\}+\|\varphi P u\|_{\frac{k-1}{m}}^{2}+R J
$$

and hence

$$
\|\varphi u\|_{0}^{2} \lesssim\|\varphi P u\|_{\frac{k-1}{m}}^{2}+R J
$$

or, iterating until $R J$ is of arbitrarily low order, for some $\tilde{\varphi} \equiv 1$ near the support of $\varphi$

$$
\|\varphi u\|_{0}^{2} \lesssim\|\tilde{\varphi} P u\|_{\frac{k-1}{m}}^{2}+\|u\|_{-N}^{2}
$$

\section{Proof of Proposition 1.3 (Optimality)}

Proof. For $\varphi$ of compact support, we set $h_{\lambda}(z, t)=\varphi v_{\lambda}$ and

$$
v_{\lambda}=\exp \left(-\lambda\left(F-i t-(F-i t)^{2}\right) .\right.
$$

If $|z|$ is small enough, we have, from above and below,

$$
\Re\left(F-i t-(F-i t)^{2}\right)=F-F^{2}+t^{2} \sim|z|^{2 m}+t^{2},
$$

and hence that $\left\|\left(\lambda\left(|z|^{2 m}+t^{2}\right)\right)^{A} v_{\lambda}\right\|_{\infty} \sim C_{A}$ uniformly in $\lambda$. Also, any function of compact support and equal to zero in a neighborhood of the origin, such as a derivative of a localizing function identically equal to one near the origin, times $v_{\lambda}$ is of order $\lambda^{-N}$ for any $N$.

Now $v_{\lambda}$ was chosen so that $\bar{L}^{F} v_{\lambda}=0$, and, letting $H=F-i t$ we compute that

$$
L_{m, k}^{F} v_{\lambda}=-2 \lambda F_{z}(1+2 H) v_{\lambda}
$$


and hence that for some $A$,

$$
\begin{aligned}
\bar{L}|z|^{2 k} L v_{\lambda} & =-2 \lambda \bar{L}\left(|z|^{2 k} F_{z}(1+2 F-2 i t)\right) v_{\lambda} \\
& \sim \lambda|z|^{2 k+2 m-2} v_{\lambda} \\
& \sim\left(\lambda|z|^{2 m}\right)^{\frac{2 k+2 m-2}{2 m}} \lambda^{1-\frac{2 k+2 m-2}{2 m}} v_{\lambda} \\
& \sim \lambda^{-\frac{k-1}{m}}\left(\lambda|z|^{2 m}\right)^{A} .
\end{aligned}
$$

Analogously, we have that as a principal term,

$$
\partial_{t}^{s} v_{\lambda} \sim \lambda^{s} v_{\lambda}
$$

Hence if there is an estimate of the form

$$
\left\|\psi v_{\lambda}\right\|_{0}^{2} \lesssim\left\|\tilde{\psi} P_{k, m}^{F} v_{\lambda}\right\|_{r}^{2}+\left\|v_{\lambda}\right\|_{-\infty}^{2}
$$

valid as $\lambda \rightarrow \infty$, for $\psi, \tilde{\psi} \in C_{0}^{\infty}, \psi \equiv 1$ near $0, \tilde{\psi} \equiv 1$ near supp $\psi$, then $r \geq \frac{k-1}{m}$, and an analogous argument holds for Proposition 1.1. Finally the optimality at all levels (other values of $s$ ) follows at once since the vector field $\partial / \partial t$ commutes with the differential operator $P_{k, m}^{F}$.

\section{First proof of Theorem 1}

As mentioned above, it is only in the 'positive cone' $\Gamma^{+}$that hypoellipticity must be shown, and then only in a small neighborhood of $z=0$. Elsewhere the operator is subelliptic in the usual sense or elliptic and (microlocal) hypoellipticity with a gain of derivatives is well known.

In $\Gamma^{+}$, since $|\zeta| \lesssim|\tau|$, showing that high derivatives in $t$ exhibit the appropriate gain will suffice. There is in general no way (as yet) to pass from an estimate such as

$$
\left\|\varphi \partial_{t}^{r} v\right\|_{s}^{2} \lesssim\left\|\tilde{\varphi} \partial_{t}^{r} P v\right\|_{s+\frac{k-1}{m}}^{2}+\|v\|_{-\infty}^{2}
$$

valid for smooth $v$ to the finiteness of $\left\|\varphi \partial_{t}^{r} u\right\|_{s}$ for a particular (distribution) solution $u$ to $P u=f$ with $\tilde{\varphi} \partial_{t}^{r} P u \in H^{s+\frac{k-1}{m}}$, however. In this situation, though, we may exploit the fact that the coefficients of $P$ do not depend on the variable $t$ and introduce a cutoff function $\chi(\tau) \equiv 1,|\tau| \leq 1$ and in $C_{0}^{\infty}(|\tau| \leq 2),|\chi| \leq 1$ and set $\chi_{M}(\tau)=\chi(\tau / M)$.

It is not difficult to see that with slight modification, the above estimate may be applied to $v=\chi_{M}\left(\partial_{t}\right) \gamma^{+} u$ where the operator $\chi_{M}\left(\partial_{t}\right)$ has the obvious meaning (via Fourier transform) and $\gamma^{+}$is supported in $\Gamma^{+}$and is equal to one near the $\tau$ axis. The slight modification is that we must add a constant $C_{u}$ independent of $M$ to handle derivatives of $\gamma^{+}$.

Thus we may write, suppressing $\gamma^{+}$,

$$
\left\|\varphi \chi_{M}\left(\partial_{t}\right) \partial_{t}^{r} u\right\|_{s}^{2} \lesssim\left\|\tilde{\varphi} \chi_{M}\left(\partial_{t}\right) \partial_{t}^{r} P u\right\|_{s+\frac{k-1}{m}}^{2}+\|u\|_{-\infty}^{2}+C_{u}
$$

and then let $M \rightarrow \infty$ to see that the previous estimate holds also for the solution $u$ and hence that $u$ is smooth in $t$ as well as in the other variables. Strictly speaking, we would need to commute $\varphi$ (or $\tilde{\varphi})$ with $\chi_{M}(\partial t$ ), intruducing a term of lower order in $\partial t$ with a derivative on the localizing function, and hence inductively handled. But this will not affect the hypoellipticity. 


\section{Second proof of Theorem $1, F(z, \bar{z})$ satisfying (1.5)}

Here we will present a second proof of the hypoellipticity of $P$ which extends naturally to a proof of analytic hypoellipticity. It was given in the model case $F(z, \bar{z})=|z|^{2 m}$ in [12].

8.1. The localization of powers of $\partial_{t}$. Localization must be done very carefully, even with $\varphi$ depending on $t$ alone. For example, the first bracket $\left[L, \varphi \partial_{t}^{p}\right]$ which we encounter will contain $(L \varphi(t)) \partial_{t}^{p} \sim i F_{z} \varphi^{\prime} \partial_{t}^{p}$, which is problematic for any value of $m$ - no gain in powers of $T$ and it is unclear even how to estimate this expression. While $\zeta$ may be small relative to $\tau$, it is not small relative to $|z|^{2 m+1} \tau$, so this error can not be estimated easily.

In Kohn's work [7] $(m=1)$, the $\bar{z}$ (or $z)$ in front of each derivative of $\varphi$ is carefully followed, and shown to provide, after some work, a gain of $1 / 2$ derivative. Analyticity was not considered in that paper, nor does is it evident that it could be shown by those methods.

Derridj and Tartakoff found in [5] that an entirely different approach, involving a delicately balanced localization of $\partial_{t}^{p}$, led to analyticity rather directly, at least for the case $F(z, \bar{z})=|z|^{2}$. Then in [12] Tartakoff proved analyticity (and $C^{\infty}$ ) hypoellipticity borrowing much of the analysis of [4] for the case $F(z, \bar{z})=|z|^{2 m}$. It is remarkable that replacing 2 by $2 m$ changes enormously the degree of technical complexity.

Here we look at $F(z, \bar{z})$ subject to the conditions described above, namely

$$
F_{z \bar{z}}=|z|^{2(m-1)} h, h(0) \neq 0, F_{z}=\bar{z}|z|^{2(m-1)} h \text { so that } F_{z}=\mu^{-1} \bar{z} F_{z \bar{z}}
$$

in the language of [4] with $\mu$ a suitable real analytic, non-zero function.

To introduce the general proof we include the short argument from [12], namely the case of $P_{k}, m=1$, i.e., $F(z, \bar{z})=|z|^{2}$.

\subsection{The case of $P_{k}$ where $m=1$.}

Definition 8.1. For any pair of non-negative integers, $\left(p_{1}, p_{2}\right)$, let

$$
T=-i \frac{\partial}{\partial t}
$$

and set

$$
\left(T_{1}^{p_{1}, p_{2}}\right)_{\varphi}=\sum_{\substack{a \leq p_{1} \\ b \leq p_{2}}} \frac{{\overline{L_{1}}}^{a} \circ \bar{z}^{a} \circ T^{p_{1}-a} \circ \varphi^{(a+b)} \circ T^{p_{2}-b} \circ z^{b} \circ L_{1}^{b}}{a ! b !},
$$

where

$$
\varphi^{(r)}=2^{-r} T^{r} \varphi
$$

Note that the leading term (with $a+b=0$ ) is merely $T^{p_{1}} \circ \varphi \circ T^{p_{2}}$ which is equal to the operator $T^{p_{1}+p_{2}}$ on any open set $\Omega_{0}$ where $\varphi \equiv 1$.

\section{Proposition 8.1.}

$$
\begin{aligned}
{\left[L_{1},\left(T_{1}^{p_{1}, p_{2}}\right)_{\varphi}\right] } & \equiv\left(T_{1}^{p_{1}, p_{2}-1}\right)_{\varphi^{\prime}} \circ L_{1}, \\
{\left[\bar{L}_{1},\left(T_{1}^{p_{1}, p_{2}}\right)_{\varphi}\right] } & \equiv \overline{L_{1}} \circ\left(T_{1}^{p_{1}-1, p_{2}}\right)_{\varphi^{\prime}}, \\
{\left[\left(T_{1}^{p_{1}, p_{2}}\right)_{\varphi}, z\right] } & =z \circ\left(T_{1}^{p_{1}, p_{2}-1}\right)_{\varphi^{\prime}},
\end{aligned}
$$


and

$$
\left[\left(T_{1}^{p_{1}, p_{2}}\right)_{\varphi}, \bar{z}\right]=\left(T_{1}^{p_{1}-1, p_{2}}\right)_{\varphi^{\prime}} \circ \bar{z}
$$

where the $\equiv$ denotes modulo $C^{p_{1}-p_{1}^{\prime}+p_{2}-p_{2}^{\prime}}$ terms of the form

$$
\frac{{\overline{L_{1}}}^{p_{1}-p_{1}^{\prime}} \circ \bar{z}^{p_{1}-p_{1}^{\prime}} \circ T^{p_{1}^{\prime}} \circ \varphi^{\left(p_{1}-p_{1}^{\prime}+p_{2}-p_{2}^{\prime}+1\right)} \circ T^{p_{2}^{\prime}} \circ z^{p_{2}-p_{2}^{\prime}} \circ L_{1}^{p_{2}-p_{2}^{\prime}}}{\left(p_{1}-p_{1}^{\prime}\right) !\left(p_{2}-p_{2}^{\prime}\right) !}
$$

with either $p_{1}^{\prime}=0$ or $p_{2}^{\prime}=0$, i.e., terms where all free $\partial_{t}$ derivatives have been eliminated on one side of $\varphi$ or the other.

Proof. The proof is a straightforward calculation involving a shift of index in the definition of $\left(T_{1}^{p_{1}, p_{2}}\right)_{\varphi}$.

As we will see below in the more general setting, these are remarkable commutation relations: each bracket will very effectively transfer one $T$ derivative onto $\varphi$ while preserving the precise balance in the localization and at the same time all the ingredients of the operator $P$ (except for the final errors, which are all in 'good' directions). And this process may be repeated until the total order in $T$ has been reduced by a factor of $3 / 4$.

8.3. The case of general $m, k$. We introduce the important vector field

$$
M=\mu^{-1} z L, \quad\left(L=L_{m}\right)
$$

that has the property

$$
[L, \bar{M}] \equiv F_{z} T \bmod \bar{M}
$$

and hence

$$
\left[L, \varphi \partial_{t}+\varphi_{t} \bar{M}\right] \equiv 0 \quad \bmod \left(L \varphi_{t}\right) \bar{M} .
$$

In other words, we have managed to kill the most disturbing term in the bracket of $L$ with $\varphi \partial_{t}$. Note that addition of $\varphi_{t} M$ would not introduce new $\partial_{t}$ upon bracketing with $L$ and yet, upon bracketing with $\bar{L}$ would also kill the most disturbing term. This suggests a relatively straightforward generalization, along the lines of [4] and [1].

Definition 8.2. For $\left(p_{1}, p_{2}\right)$ as above, set

$$
\varphi^{(d)}=\left(i \partial_{t}\right)^{d} \varphi(t)
$$

and set

$$
N_{b}=\sum_{b^{\prime} \leq b} A_{b^{\prime}}^{b} \frac{M^{b^{\prime}}}{b^{\prime} !}
$$

where the $A_{b^{\prime}}^{b}$ (real) are to be determined subject to $A_{b}^{b}=1$, and

$$
\tilde{N}_{a}=\sum_{a^{\prime} \leq a} A_{a^{\prime}}^{a} \frac{M^{* a^{\prime}}}{a^{\prime} !}=\left(N_{a}\right)^{*}
$$

where $M^{*}=-\bar{L} \circ \bar{z} \circ \mu^{-1}$, and set

$$
\left(T^{p_{1}, p_{2}}\right)_{\varphi}=\sum_{\substack{a \leq p_{1} \\ b \leq p_{2}}} \tilde{N}_{a} \circ T^{p_{1}-a} \circ \varphi^{(a+b)} \circ T^{p_{2}-b} \circ N_{b}
$$


We have

$$
\begin{aligned}
{\left[\bar{L},\left(T^{p_{1}, p_{2}}\right)_{\varphi}\right]=} & {\left[\bar{L}, \sum_{a \leq p_{1}, b \leq p_{2}} \tilde{N}_{a} T^{p_{1}-a} \varphi^{(a+b)} T^{p_{2}-b} N_{b}\right] } \\
= & \sum_{a \leq p_{1}, b \leq p_{2}}\left\{\left[\bar{L}, \tilde{N}_{a}\right] T^{p_{1}-a} \varphi^{(a+b)} T^{p_{2}-b} N_{b}\right. \\
& \left.-\tilde{N}_{a} T^{p_{1}-a} F_{\bar{z}} \varphi^{(a+b+1)} T^{p_{2}-b} N_{b}+\tilde{N}_{a} T^{p_{1}-a} \varphi^{(a+b)} T^{p_{2}-b}\left[\bar{L}, N_{b}\right]\right\} .
\end{aligned}
$$

The last two terms on the right must cancel, to preserve the balance, since both disturb the balance between derivatives on $\varphi$ and gain in powers of $T$. We will choose the coefficients $A_{b^{\prime}}^{b}$ of $N_{b}$ in such a way that, modulo acceptable errors,

$$
\left[\bar{L}, N_{b}\right]=-F_{\bar{z}} T N_{b-1} .
$$

This will provide the needed cancellation via a shift of index in $b$ in the sum just as in the case with $F=|z|^{2}$. The corresponding relation for brackets with $L$ will follow by taking adjoints: again modulo acceptable errors,

$$
\left[L, \tilde{N}_{a}\right]=-\tilde{N}_{a-1} F_{z} T .
$$

Condition (8.5) reads, using the definition of $N_{b}$, reads:

$$
\sum_{b^{\prime}=0}^{b} A_{b^{\prime}}^{b} \frac{1}{b^{\prime} !}\left[\bar{L}, M^{b^{\prime}}\right]=-F_{\bar{z}} T \sum_{b^{\prime}=0}^{b-1} A_{b^{\prime}}^{b-1} \frac{M^{b^{\prime}}}{b^{\prime} !} .
$$

Expanding the brackets and keeping all factors of $z L$ to the right,

$$
\begin{aligned}
\frac{1}{b^{\prime} !}\left[\bar{L}, M^{b^{\prime}}\right] & =-\frac{1}{b^{\prime} !} \sum_{1 \leq b^{\prime \prime} \leq b^{\prime}}\left(\begin{array}{c}
b^{\prime} \\
b^{\prime \prime}
\end{array}\right) a d_{M}^{b^{\prime \prime}}(\bar{L}) M^{b^{\prime}-b^{\prime \prime}} \\
& =-\frac{1}{b^{\prime} !} \sum_{1 \leq b^{\prime \prime} \leq b^{\prime}}\left(\begin{array}{c}
b^{\prime} \\
b^{\prime \prime}
\end{array}\right) a d_{M}^{b^{\prime \prime}-1}\left(F_{\bar{z}} T\right) M^{b^{\prime}-b^{\prime \prime}} \\
& =-\left(F_{\bar{z}} T\right) \sum_{1 \leq b^{\prime \prime} \leq b^{\prime}} \frac{1}{b^{\prime \prime} !} \frac{1}{2^{b^{\prime \prime}-1}} \frac{M^{b^{\prime}-b^{\prime \prime}}}{\left(b^{\prime}-b^{\prime \prime}\right) !}
\end{aligned}
$$

since $M F_{\bar{z}}=2^{-1} F_{\bar{z}}$. The condition (8.5) thus requires, renaming $b^{\prime}-b^{\prime \prime}$ as $\tilde{b}$ on the right just above,

$$
\sum_{b^{\prime \prime}=1}^{b-\tilde{b}} A_{\tilde{b}+b^{\prime \prime}}^{b} \frac{1}{b^{\prime \prime} !} \frac{1}{2^{b^{\prime \prime}-1}}=A_{\tilde{b}}^{b-1} .
$$

Fortunately, these equations have been investigated in [4] and, using a result in the book by Hirzebruch [6] have explicit solutions $\widetilde{A}_{*}^{*}$, unique under the conditions that $\tilde{A}_{0}^{q}=(-1)^{q} 2^{q}$, namely

$$
2^{r-s} \tilde{A}_{s}^{r}=\left(\left(\frac{t}{e^{t}-1}\right)^{r+1}\right)^{(r-s)}(0) /(r-s) !
$$


In addition, we will also need good expressions for the other brackets: we compute

$$
\begin{gathered}
{\left[L, N_{b}\right]=\left[L, \sum_{b^{\prime}=0}^{b} A_{b^{\prime}}^{b} \frac{M^{b^{\prime}}}{b^{\prime} !}\right]=\sum_{\substack{1 \leq b^{\prime \prime} \leq b^{\prime} \\
b^{\prime} \leq b}} A_{b^{\prime}}^{b} \frac{1}{b^{\prime \prime} !} \frac{1}{2^{b^{\prime \prime}}} \frac{M^{b^{\prime}-b^{\prime \prime}}}{\left(b^{\prime}-b^{\prime \prime}\right) !} \circ L} \\
{\left[N_{b}, z\right]=z \circ \sum_{\substack{1 \leq b^{\prime \prime} \leq b^{\prime} \\
b^{\prime} \leq \bar{b}}} A_{b^{\prime}}^{b} \frac{1}{b^{\prime \prime} !} \frac{1}{2^{b^{\prime \prime}}} \frac{M^{b^{\prime}-b^{\prime \prime}}}{\left(b^{\prime}-b^{\prime \prime}\right) !}} \\
{\left[\bar{L}, \tilde{N}_{a}\right]=-\bar{L} \circ \sum_{\substack{1 \leq a^{\prime \prime} \leq a^{\prime} \\
a^{\prime} \leq a}} A_{a^{\prime}}^{a} \frac{1}{a^{\prime \prime !} !} \frac{1}{2^{a^{\prime \prime}}} \frac{\left(M^{*}\right)^{a^{\prime}-a^{\prime \prime}}}{\left(a^{\prime}-a^{\prime \prime}\right) !},} \\
{\left[\bar{z}, \tilde{N}_{a}\right]=\sum_{\substack{1 \leq a^{\prime \prime} \leq a^{\prime} \\
a^{\prime} \leq a}} A_{a^{\prime}}^{a} \frac{1}{a^{\prime \prime} !} \frac{1}{2^{a^{\prime \prime}}} \frac{\left(M^{*}\right)^{a^{\prime}-a^{\prime \prime}}}{\left(a^{\prime}-a^{\prime \prime}\right) !} \circ \bar{z} .}
\end{gathered}
$$

In order to recognize these sums as $N$ 's or $\tilde{N}$ 's, we need to be able to shift the lower indices on $A_{a^{\prime}}^{a}$ down by one. But this also we have done in [4], with the result that

Proposition 8.2. For any $r, s$, and $c$,

$$
A_{s}^{r}=\sum_{j=0}^{r-s} S_{j}^{c+j} 2^{-j} A_{s-c}^{r-(c+j)}
$$

where

$$
\left|S_{\ell}^{k}\right| \leq C^{k}
$$

These brackets, then, together with the Proposition, immediately translate, setting $b^{\prime \prime}=c$ and $\tilde{b}=b^{\prime}-b^{\prime \prime}$, into:

or

$$
\left[L, N_{b}\right]=\sum_{\tilde{b} \leq b-c} \frac{1}{c !} \frac{1}{2^{c}} A_{\tilde{b}+c}^{b} \frac{M^{\tilde{b}}}{\tilde{b} !} \circ L=\sum_{\tilde{b} \leq b-c-j} \frac{1}{c !} \frac{1}{2^{c}} S_{j}^{c+j} A_{\tilde{b}}^{b-c-j} \frac{M^{\tilde{b}}}{\tilde{b} !} \circ L
$$

$$
\left[L, N_{b}\right]=\sum_{\substack{c+j \leq b \\ 1 \leq c}} \frac{1}{c !} \frac{1}{2^{c}} S_{j}^{c+j} N_{b-c-j} \circ L
$$

Similarly,

$$
\left[\bar{L}, \tilde{N}_{a}\right]=-\bar{L} \circ \sum_{\substack{c+j \leq a \\ 1 \leq c}} \frac{1}{c !} \frac{1}{2^{c}} S_{j}^{c+j} \tilde{N}_{a-c-j}
$$

and

$$
\left[\bar{z}, \tilde{N}_{a}\right]=\sum_{\substack{c+j \leq a \\ 1 \leq c}} \frac{1}{c !} \frac{1}{2^{c}} S_{j}^{c+j} \tilde{N}_{a-c-j} \circ \bar{z}
$$

and

$$
\left[N_{b}, z\right]=z \circ \sum_{\substack{c+j \leq b \\ 1 \leq c}} \frac{1}{c !} \frac{1}{2^{c}} S_{j}^{c+j} \tilde{N}_{b-c-j}
$$


These precise commutation relations mean that the whole localization $\left(T^{p_{1}, p_{2}}\right)_{\varphi}$ may be commuted meaningfully with the vector fields $L, \bar{L}$ and with $z, \bar{z}$ :

Proposition 8.3. Modulo terms in which either $p_{1}$ or $p_{2}$ has been reduced to zero, and in view of the cancellations ensured by (8.5),

$$
\left[L,\left(T^{p_{1}, p_{2}}\right)_{\varphi}\right] \equiv \sum_{\substack{1 \leq c, 0 \leq j \\ c+j \leq p_{2}}} \frac{1}{c !} \frac{1}{2^{c}} S_{j}^{c+j}\left(T^{p_{1}, p_{2}-(c+j)}\right)_{\varphi^{(c+j)}} \circ L
$$

Proof.

$$
\begin{gathered}
{\left[L,\left(T^{p_{1}, p_{2}}\right)_{\varphi}\right] \equiv \sum_{\substack{a \leq p_{1} \\
b \leq p_{2}}} \tilde{N}_{a} \circ T^{p_{1}-a} \circ \varphi^{(a+b)} \circ T^{p_{2}-b}\left[L, N_{b}\right]} \\
\equiv \sum_{\substack{a \leq p_{1} \\
b \leq p_{2}}} \sum_{\substack{c+j \leq b \\
1 \leq c}} \frac{1}{c !} \frac{1}{2^{c}} S_{j}^{c+j} \tilde{N}_{a} \circ T^{p_{1}-a} \circ \varphi^{(a+b)} \circ T^{p_{2}-b} N_{b-c-j} \circ L \\
\equiv \sum_{\substack{a \leq p_{1} \\
b \leq p_{2}}} \sum_{\substack{c+j \leq b \\
1 \leq c}} \frac{1}{c !} \frac{1}{2^{c}} S_{j}^{c+j} \tilde{N}_{a} T^{p_{1}-a} \varphi^{(c+j)^{(a+b-c-j)}} T^{p_{2}-(c+j)-(b-c-j)} N_{b-c-j} \circ L \\
\equiv \sum_{\substack{1 \leq c, 0 \leq j \\
c+j \leq p_{2}}} \frac{1}{c !} \frac{1}{2^{c}} S_{j}^{c+j}\left(T^{p_{1}, p_{2}-(c+j)}\right)_{\varphi^{(c+j)}} \circ L
\end{gathered}
$$

Similarly we state, and omit the proofs, which are virtually identical to that of the previous proposition,

\section{Proposition 8.4.}

$$
\begin{aligned}
{\left[\bar{L},\left(T^{p_{1}, p_{2}}\right)_{\varphi}\right] } & \equiv-\bar{L} \circ \sum_{\substack{1 \leq c, 0 \leq j \\
c+j \leq p_{1}}} \frac{1}{c !} \frac{1}{2^{c}} S_{j}^{c+j}\left(T^{p_{1}-(c+j), p_{2}}\right)_{\varphi^{(c+j)}}, \\
{\left[\bar{z},\left(T^{p_{1}, p_{2}}\right)_{\varphi}\right] } & \equiv \sum_{\substack{1 \leq c, 0 \leq j \\
c+j \leq p_{1}}} \frac{1}{c !} \frac{1}{2^{c}} S_{j}^{c+j}\left(T^{p_{1}-(c+j), p_{1}}\right)_{\varphi^{(c+j)}} \circ \bar{z}
\end{aligned}
$$

and

$$
\left[z,\left(T^{p_{1}, p_{2}}\right)_{\varphi}\right] \equiv-\sum_{\substack{1 \leq c, 0 \leq j \\ c+j \leq p_{2}}} \frac{1}{c !} \frac{1}{2^{c}} S_{j}^{c+j}\left(T^{p_{1}, p_{2}-(c+j)}\right)_{\varphi^{(c+j)}} \circ z
$$

What these commutation relations mean is that we may move the vector fields of $P_{m}$ past $\left(T^{p_{1}, p_{2}}\right)_{\varphi}$ freely, at each stage incurring errors with the same vector fields and a gain in derivatives in $\left(T^{p_{1}, p_{2}}\right)_{\varphi}$. Thus we may iterate the a priori inequality modulo errors of nearly arbitrarily low order - all of the $\equiv$ signs above mean that we will ultimately arrive at errors where either $p_{1}=0$ or $p_{2}=0$.

So we insert first $v=\left(T^{p_{1}, p_{2}}\right)_{\varphi} u$ into $(1.7)$, then bring $\left(T^{p_{1}, p_{2}}\right)_{\varphi}$ to the left of $P=L \bar{L}+\bar{L} z^{k} \bar{z}^{k} L$, and find that we have:

$$
\left\|\bar{L}\left(T^{p_{1}, p_{2}}\right)_{\varphi} u\right\|_{0}^{2}+\left\|\bar{z}^{k} L\left(T^{p_{1}, p_{2}}\right)_{\varphi} u\right\|_{0}^{2}+\left\|\Lambda^{-\frac{k-1}{2}}\left(T^{\frac{p}{2}, \frac{p}{2}}\right)_{\varphi} u\right\|_{0}^{2}
$$




$$
\begin{gathered}
\lesssim\left|\left(P\left(T^{p_{1}, p_{2}}\right)_{\varphi} u,\left(T^{p_{1}, p_{2}}\right)_{\varphi} u\right)_{L^{2}}\right| \\
\lesssim\left|\left(\left(T^{p_{1}, p_{2}}\right)_{\varphi} P u,\left(T^{p_{1}, p_{2}}\right)_{\varphi} u\right)_{L^{2}}\right|+\left|\left(\left[P,\left(T^{p_{1}, p_{2}}\right)_{\varphi}\right] u,\left(T^{p_{1}, p_{2}}\right)_{\varphi} u\right)_{L^{2}}\right|
\end{gathered}
$$

and by the above bracket relations, modulo the same terms as above where all $T$ 's from one side of $\varphi$ or the other have been 'converted' into $L$ 's or $\bar{L}$ 's, we have

$$
\begin{aligned}
& \left(\left[P,\left(T^{p_{1}, p_{2}}\right)_{\varphi}\right] u,\left(T^{p_{1}, p_{2}}\right)_{\varphi} u\right) \equiv \\
& =\left(\left[L \bar{L},\left(T^{p_{1}, p_{2}}\right)_{\varphi}\right] u,\left(T^{p_{1}, p_{2}}\right)_{\varphi} u\right)+\left(\left[\bar{L} z^{k} \bar{z}^{k} L,\left(T^{p_{1}, p_{2}}\right)_{\varphi}\right] u,\left(T^{p_{1}, p_{2}}\right)_{\varphi} u\right) \\
& =\left(\left[L,\left(T^{p_{1}, p_{2}}\right)_{\varphi}\right] \bar{L} u,\left(T^{p_{1}, p_{2}}\right)_{\varphi} u\right)+\left(L\left[\bar{L},\left(T^{p_{1}, p_{2}}\right)_{\varphi}\right] u,\left(T^{p_{1}, p_{2}}\right)_{\varphi} u\right) \\
& +\left(\left[\bar{L},\left(T^{p_{1}, p_{2}}\right)_{\varphi}\right] z^{k} \bar{z}^{k} L u,\left(T^{p_{1}, p_{2}}\right)_{\varphi} u\right)+\left(\bar{L}\left[z^{k},\left(T^{p_{1}, p_{2}}\right)_{\varphi}\right] \bar{z}^{k} L u,\left(T^{p_{1}, p_{2}}\right)_{\varphi} u\right) \\
& +\left(\bar{L} z^{k}\left[\bar{z}^{k},\left(T^{p_{1}, p_{2}}\right)_{\varphi}\right] L u,\left(T^{p_{1}, p_{2}}\right)_{\varphi} u\right)+\left(\bar{L} z^{k} \bar{z}^{k}\left[L,\left(T^{p_{1}, p_{2}}\right)_{\varphi}\right] u,\left(T^{p_{1}, p_{2}}\right)_{\varphi} u\right) \\
& \equiv \sum_{\substack{1 \leq c, 0 \leq j \\
c+j \leq p_{2}}} \frac{1}{c !} \frac{1}{2^{c}} S_{j}^{c+j}\left(\left(T^{p_{1}, p_{2}-(c+j)}\right)_{\varphi^{(c+j)}} L \bar{L} u,\left(T^{p_{1}, p_{2}}\right)_{\varphi} u\right) \\
& -\sum_{\substack{1 \leq c, 0 \leq j \\
c+j \leq p_{1}}} \frac{1}{c !} \frac{1}{2^{c}} S_{j}^{c+j}\left(L \bar{L}\left(T^{p_{1}-(c+j), p_{2}}\right)_{\varphi(c+j)} u,\left(T^{p_{1}, p_{2}}\right)_{\varphi} u\right) \\
& -\sum_{\substack{1 \leq c, 0 \leq j \\
c+j \leq p_{1}}} \frac{1}{c !} \frac{1}{2^{c}} S_{j}^{c+j}\left(\bar{L}\left(T^{p_{1}-(c+j), p_{2}}\right)_{\varphi^{(c+j)}} z^{k} \bar{z}^{k} L u,\left(T^{p_{1}, p_{2}}\right)_{\varphi} u\right) \\
& -\sum_{k^{\prime}=1}^{k} \sum_{\substack{1 \leq c, 0 \leq j \\
c+j \leq p_{2}}} \frac{1}{c !} \frac{1}{2^{c}} S_{j}^{c+j}\left(\bar{L} z^{k^{\prime}}\left(T^{p_{1}, p_{2}-(c+j)}\right)_{\varphi^{(c+j)}} z^{k-k^{\prime}} \bar{z}^{k} L u,\left(T^{p_{1}, p_{2}}\right)_{\varphi} u\right) \\
& +\sum_{k^{\prime}=0}^{k-1} \sum_{\substack{1 \leq c, 0 \leq j \\
c+j \leq p_{1}}} \frac{1}{c !} \frac{1}{2^{c}} S_{j}^{c+j}\left(\bar{L} z^{k} \bar{z}^{k^{\prime}}\left(T^{p_{1}-(c+j), p_{1}}\right)_{\varphi(c+j)} \bar{z}^{k-k^{\prime}} L u,\left(T^{p_{1}, p_{2}}\right)_{\varphi} u\right) \\
& +\sum_{\substack{1 \leq c, 0 \leq j \\
c+j \leq p_{2}}} \frac{1}{c !} \frac{1}{2^{c}} S_{j}^{c+j}\left(\bar{L} z^{k} \bar{z}^{k}\left(T^{p_{1}, p_{2}-(c+j)}\right)_{\varphi^{(c+j)}} L u,\left(T^{p_{1}, p_{2}}\right)_{\varphi} u\right) \\
& =A_{1}+A_{2}+A_{3}+\sum_{k^{\prime}=1}^{k} A_{4, k^{\prime}}+\sum_{k^{\prime}=0}^{k-1} A_{5, k^{\prime}}+A_{6} .
\end{aligned}
$$

Concerning the critical $L, \bar{L}, z^{k}$ and $\bar{z}^{k}$, note that in each term above

- no $L, \bar{L}$, power of $z$ or power of $\bar{z}$ has been lost,

- the order among $L, \bar{z}^{k}, z^{k}$, and $\bar{L}$ is preserved,

- letting $|q|=q_{1}+q_{2}$, each term on the right contains $\left(T^{q_{1}, q_{2}}\right)_{\varphi(|p|-|q|)}$ with $|q|<|p|$ and $|p|-|q|$ derivatives on $\varphi$,

- just as (8.14) demonstrates the errors which result in moving $\left(T^{p_{1}, p_{2}}\right)_{\varphi}$ past the vector fields $L, \bar{L}, z^{k} \bar{L}$ and $\bar{z}^{k} L$, further such brackets to position the vector fields so as to make use the a priori estimate again will produce similar errors, with $|q|$ still lower and the 'lost' $T$ derivatives transferred to $\varphi$,

- iterating this process, together with a weighted Schwarz inequality, will produce a sum of terms with $q_{j} \leq \frac{p}{2}$ of the form

$$
\text { l.c. } \left.\left\|\Lambda^{\frac{k-1}{2 m}}\left(T^{q_{1}, q_{2}}\right)_{\varphi(|p|-|q|)} P u\right\|_{0}^{2}+\text { s.c. } \| \Lambda^{-\frac{k-1}{2 m}}\left(T^{\frac{p}{2}, \frac{p}{2}}\right)_{\varphi} u\right) \|_{0}^{2} .
$$


- In fact, using larger constants $\tilde{S}_{j}^{c+j}$ subject to the same kind of bounds, $\left|\tilde{S}_{j}^{c+j}\right| \leq \tilde{C}^{c+j}$, we may replace all sums on the right hand side above by suprema subject to the same range restrictions on the indices.

- This use of suprema allows us easily to iterate everything on the right with easy control on the constants until either $p_{1}$ or $p_{2}$, both of which start as $\frac{p}{2}$, drops to zero, which may happen in two ways - either by stepwise decrease as on the right hand side above from successive brackets or by the single term in Propositions 8.1 and 8.3 which is not cancelled, the term with all $L$ 's or $\bar{L}$ 's on one side or the other in the definition of $\left(T^{p_{1}, p_{2}}\right)_{\varphi}$, whose principal term is $\left(T^{p_{1}, 0}\right)_{\varphi\left(p_{2}\right)}(z L)^{p_{2}} / p_{2}$ ! or its analogue with $p_{1}$ reduced to 0 .

- At this point we no longer have an effective localization of powers of $T$ - for example, brackets with $\bar{L}$ are not corrected. We proceed anyway, and when we lack a 'good' vector field such as $\bar{L}$ (or of course $\bar{z}^{k} L$ ), we create one by integrating by parts:

$$
\|L w\|_{0}^{2} \lesssim\|\bar{L} w\|_{0}^{2}+\left|\left(|z|^{2(m-1)} T w, w\right)\right|
$$

to use up the $L$ and $\bar{L}$ derivatives with the byproduct of introducing up to half the number of new $T$ derivatives.

Overall, then, the strategy has been:

$$
\begin{aligned}
\left\|\Lambda^{-\frac{k-1}{2 m}} T^{p} u\right\|_{\{\varphi \equiv 1\}}^{2} & \rightarrow\left\|\Lambda^{-\frac{k-1}{2 m}}\left(T^{\frac{p}{2}, \frac{p}{2}}\right)_{\varphi} u\right\|^{2} \rightarrow \\
\rightarrow C^{p / 4}\left\|\Lambda^{-\frac{k-1}{2 m}} T^{\frac{3 p}{4}} u\right\|_{\text {supp } \varphi}^{2} & \rightarrow C^{p / 4}\left\|\Lambda^{-\frac{k-1}{2 m}} T^{\frac{3 p}{4}} u\right\|_{\left\{\varphi_{1} \equiv 1\right\}}^{2} \rightarrow \ldots
\end{aligned}
$$

for suitable $\varphi_{1} \equiv 1$ on the support of $\varphi$. This will continue, with a sequence of $\varphi_{j}$ supported in nested open sets as in [10], [11] until only a negligible fraction of $p$ is left, namely a bounded number of derivatives. Since the order in $T^{p}$ is reduced by a factor of $3 / 4$ each time, we will need $\log _{4 / 3} p$ such nested open sets. Thus (where the constant $C_{P u}^{\left(p+\frac{k-1}{2 m}\right)}$ will reflect bounds on the derivatives of $\left.P u\right)$ :

$$
\left\|\Lambda^{-\frac{k-1}{2 m}} T^{p} u\right\|_{\{\varphi \equiv 1\}}^{2} \lesssim C^{p}\left\|\Lambda^{-\frac{k-1}{2 m}+3} u\right\|_{\left\{\varphi_{\log _{4 / 3} p} \equiv 1\right\}}^{2}+C_{P u}^{\left(p+\frac{k-1}{2 m}\right)}
$$

where the 3 could be any other small integer. And of course the whole derivation could have been done at the $H^{s}$ level: for any given $s$,

$$
\left\|\Lambda^{s-\frac{k-1}{2 m}} T^{p} u\right\|_{\{\varphi \equiv 1\}}^{2} \lesssim C_{s}^{p}\left\|\Lambda^{s-\frac{k-1}{2 m}+3} u\right\|_{\left\{\varphi_{\log _{4 / 3} p} \equiv 1\right\}}^{2}+C_{s, P u}^{\left(p+\frac{k-1}{2 m}\right)}
$$

which will end the story if this last norm is known to be finite, provided that the terms that arise along the way are all similarly bounded. The most important of these is of course

$$
\left|\left(\Lambda^{s+\frac{k-1}{2 m}}\left(T^{\frac{p}{2}, \frac{p}{2}}\right)_{\varphi} P u, \Lambda^{s-\frac{k-1}{2 m}}\left(T^{\frac{p}{2}, \frac{p}{2}}\right)_{\varphi} u\right)\right|
$$

which shows that $P u \in H^{s+\frac{k-1}{2 m}+p}$ in the largest of the nested open sets implies that $u \in H^{s-\frac{k-1}{2 m}+p}$ in the smallest, a loss of $k-1$ derivatives.

The value of $s$ will be chosen so that we know the norm on the right in (8.18) is finite (for every distribution is locally in some $H^{\tilde{s}}$ ), and then $p$ will be chosen so that $P u \in$ $H^{s+\frac{k-1}{2 m}+p}\left(\cup \operatorname{supp} \varphi_{j}\right)$ for that value of $s$. It follows that $u \in H^{s-\frac{k-1}{2 m}+p}\left(\cap \operatorname{supp} \varphi_{j}\right)$.

The calculations above were actually carried out in [4] for the case $F(z, \bar{z})=f\left(|z|^{2}\right)$, the crucial property, even for non-constant $\mu$, being that $M F_{\bar{z}}=F_{\bar{z}} / 2$, which is what 
we have used above. The relations in the Propositions (8.2,), (8.3) and (8.4) above retain their principal terms, with the same values of $A_{b^{\prime}}^{b}$ and $S_{k}^{j}$, and with lower order terms of the same form which yield to iteration as here. Finally, $F(z, \bar{z})$ of the generality treated in obtaining the estimates involves in addition, commutators with general functions, again treated in [4] and [10], [11], whose details we omit.

Remark 1. For analyticity, one needs to ensure that as we take $p$ larger and larger, the constants satisfied by the Ehrenpreis-type localizers are subject to bounds such that the estimate (8.16) is uniform in p. This has been shown often before (cf. [10], [11]) and the arguments are the same here.

\section{References}

[1] A. Bove and D.S. Tartakoff, Analytic hypo-ellipticity at non-symplectic characteristics when the symplectic form changes its rank, in preparation.

[2] _ Optimal non-isotropic Gevrey exponents for sums of squares of vector fields, Comm. Partial Differential Equations 22 (1997) 1262-1282.

[3] M. Christ, A remark on sums of squares of complex vector fields, arXiv:math.CV/0503506.

[4] M. Derridj and D.S. Tartakoff, Local analyticity for $\square_{b}$ and the $\bar{\partial}$-Neumann problem at certain weakly pseudoconvex points, Comm. Partial Differential Equations 13 (1988) 1521-1600.

[5] _ Analyticity and loss of derivatives, Ann. of Math. (2) 162 (2005) 982-986.

[6] F. Hirzebruch, Topological Methods in Algebraic Geometry, Springer Verlag, New York, 1966.

[7] J.J. Kohn, Hypoellipticity and loss of derivatives, Ann. of Math. (2) 162 (2005) 943-982.

[8] C. Parenti and A. Parmeggiani, A note on Kohn's and Christ's Examples, preprint.

[9] Y.-T. Siu,, Extension of twisted pluricanonical sections with plurisubharmonic weight and invariance of semipositively twisted plurigenera for manifolds not necessarily of general type, in Complex Geometry: Collection of Papers Dedicated to Professor Hans Grauert, 223-277, Springer-Verlag, New York, 2002.

[10] D.S. Tartakoff, Local analytic hypoellipticity for $\square_{b}$ on non-degenerate Cauchy Riemann manifolds, Proc. Nat. Acad. Sci. U.S.A. 75 (1978) 3027-03028.

[11] , On the local real analyticity of solutions to $\square_{b}$ and the $\bar{\partial}$-Neumann problem, Acta Math. 145 (1980) 117-204.

[12] _ Analyticity for singular sums of squares of degenerate vector fields, to appear, Proc. Amer. Math. Soc. 2005.

[13] F. Treves, Analytic hypo-ellipticity of a class of pseudo-differential O(perators with double characteristics and application to the $\bar{\partial}-$ Neumann problem, Comm. Partial Differential Equations 3 (1978) 475-642.

Dipartimento di Matematica, Università di Bologna, Piazza di Porta San Donato 5 , BOLOGNA ITALY

E-mail address: bove@dm.unibo.it

5 Rue de la Juvinière, 78350 Les Loges en Josas, FRANCE

E-mail address: maklouf.derridj@wanadoo.fr

Department of Mathematics, Fine Hall, Princeton University, Princeton NJ 08540

E-mail address: kohn@math.princeton.edu

Department of Mathematics, University of Illinois at Chicago, m/C 249, 851 S. Morgan St., Chicago IL 60607

E-mail address: dst@uic.edu 